\title{
Solid-State Analysis of Alpha-Cyclodextrin Inclusion Complexes Using Low-Frequency Raman Spectroscopy
}

Motoki Inoue,${ }^{* \dagger}$ Hiroshi Hisada, ${ }^{\dagger}$ Kazuhiko Takatori,${ }^{\dagger}$ Tatsuo Koide, ${ }^{\dagger}$ Toshiro Fukami, ${ }^{\dagger}$ Anjan Roy, ${ }^{\S}$ James Carriere, ${ }^{\S}$

$\dagger$ Meiji Pharmaceutical University, 2-522-1, Noshio, Kiyose, Tokyo 204-8588, Japan

National Institute of Health Sciences, 3-25-26, Tonomachi, Kawasaki-ku, Kawasaki, Kanagawa 210-9501, Japan

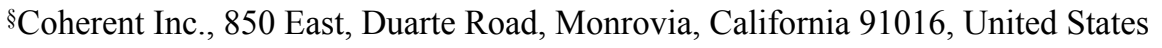

- X-ray Crystallographic Analysis

Table of Contents

References 


\section{X-ray Crystallographic Analysis}

X-ray crystallographic data were recorded on a Rigaku R-AXIS RAPID with a MicroMax-007HF diffractometer using multi-layer mirror monochromated $\mathrm{Cu}-\mathrm{K} \alpha$ radiation. The structure was solved by a direct method (SHELXT ver. 2014/5) ${ }^{1}$ and expanded using a Fourier technique. Refinement was performed using all reflections. All calculations were performed using the CrystalStructure 4.2.4 crystallographic software package except for refinement, which was performed using SHELXL ver. 2014/7.2

\section{$\alpha$-CD-4-nitrophenol complex}

The non-hydrogen atoms were refined anisotropically. The hydrogen atoms other than waters were refined using the riding model. The hydrogen atoms bonded to non-disordered water were could located on a difference Fourier synthesis, however the hydrogen atoms bonded to disordered waters could not be located.
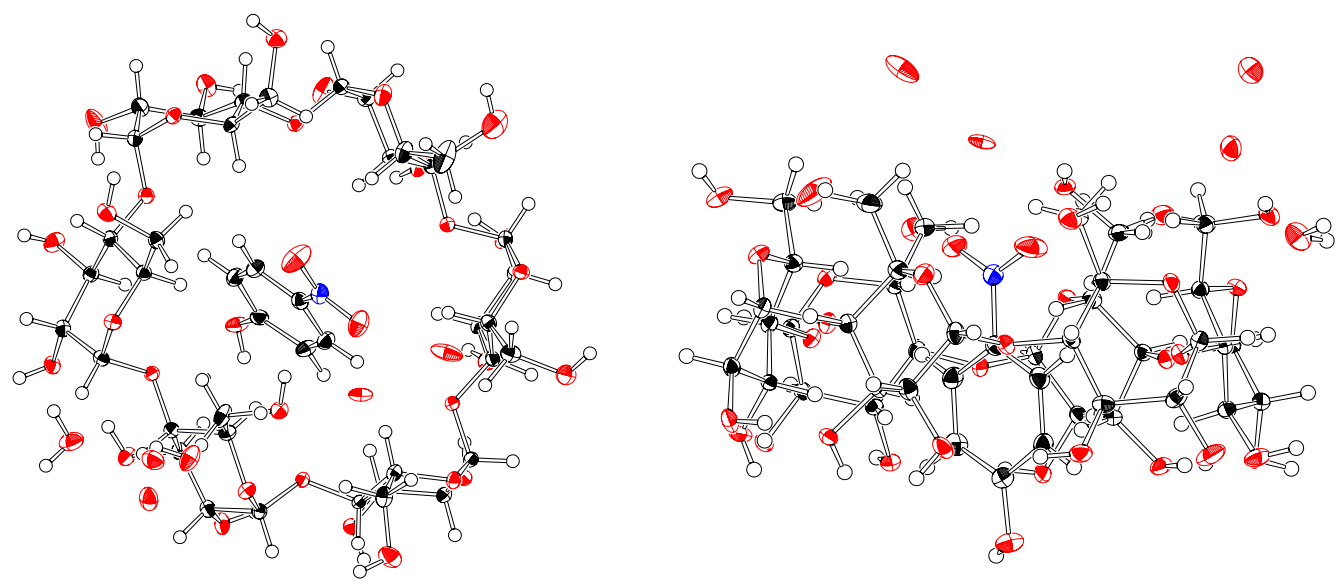

ORTEP drawing of $\alpha$-CD-4-nitrophenol complex 
Crystal data for $\alpha$-CD-4-nitrophenol complex

$\begin{array}{ll}\text { Chemical formula } & \mathrm{C}_{42} \mathrm{H}_{67} \mathrm{NO}_{36} \\ \text { Moiety formula } & \mathrm{C}_{36} \mathrm{H}_{60} \mathrm{O}_{30}, \mathrm{C}_{6} \mathrm{H}_{5} \mathrm{NO}_{3}, \mathrm{H}_{2} \mathrm{O}, 2(\mathrm{O}) \\ \text { MW } & 1161.97 \\ \text { Crystal system, space group } & \text { Orthorhombic, } \mathrm{P}_{1}{ }_{2} 2_{1} \\ \text { Temperature }(\mathrm{K}) & 93 \\ a, b, c(\AA), V\left(\AA^{3}\right) & 13.4340(3), 15.1823(3), 24.7053(5), 5038.87(18) \\ Z & 4 \\ F(000) & 2456.00 \\ \text { Radiation type } & \mathrm{Cu} K \alpha(\lambda=1.54187 \AA) \\ \mu\left(\mathrm{mm}{ }^{-1}\right) & 1.19 \\ \text { Crystal size }(\mathrm{mm}), \text { and color } & 0.22 \times 0.20 \times 0.18, \text { prism, colorless }\end{array}$

Data collection

Diffractometer

Absorption correction

\section{Rigaku R-AXIS RAPID}

Multi-scan

ABSCOR (Rigaku, 1995)

$T_{\min }, T_{\max }$

$0.680,0.807$

No. of measured, independent, and

observed $\left[F^{2}>2.0 \sigma\left(F^{2}\right)\right]$ reflections

$59282,9182,9120$

$R_{\text {int }}$

0.022

$(\sin \theta / \lambda)_{\max }\left(\AA^{-1}\right)$

0.6017

Refinement

$\mathrm{R}\left[F^{2}>2 \sigma\left(F^{2}\right)\right], w R\left(F^{2}\right), S$

$0.034,0.094,1.05$

No. of reflections

9182

No. of parameters

765

H-atom treatment

$\mathrm{H}$ atoms treated by a mixture of independent and constrained refinement

$\Delta \rho_{\max }, \Delta \rho_{\min }\left(\mathrm{e} \AA^{-3}\right)$

$0.66,-0.52$

Flack x parameter ${ }^{3}$ (Parsons quotients $=4013$ )

$0.041(14)$ 


\section{a-CD-4-iodophenol complex}

The non-hydrogen atoms and the non-disordered oxygen atoms were refined anisotropically, and the disordered oxygen atoms with high and low occupancy were refined anisotropically and isotropically, respectively. The hydrogen atoms other than waters were refined using the riding model. The hydrogen atoms bonded to non-disordered water were could located on a difference Fourier synthesis, however the hydrogen atoms bonded to disordered waters were not located.
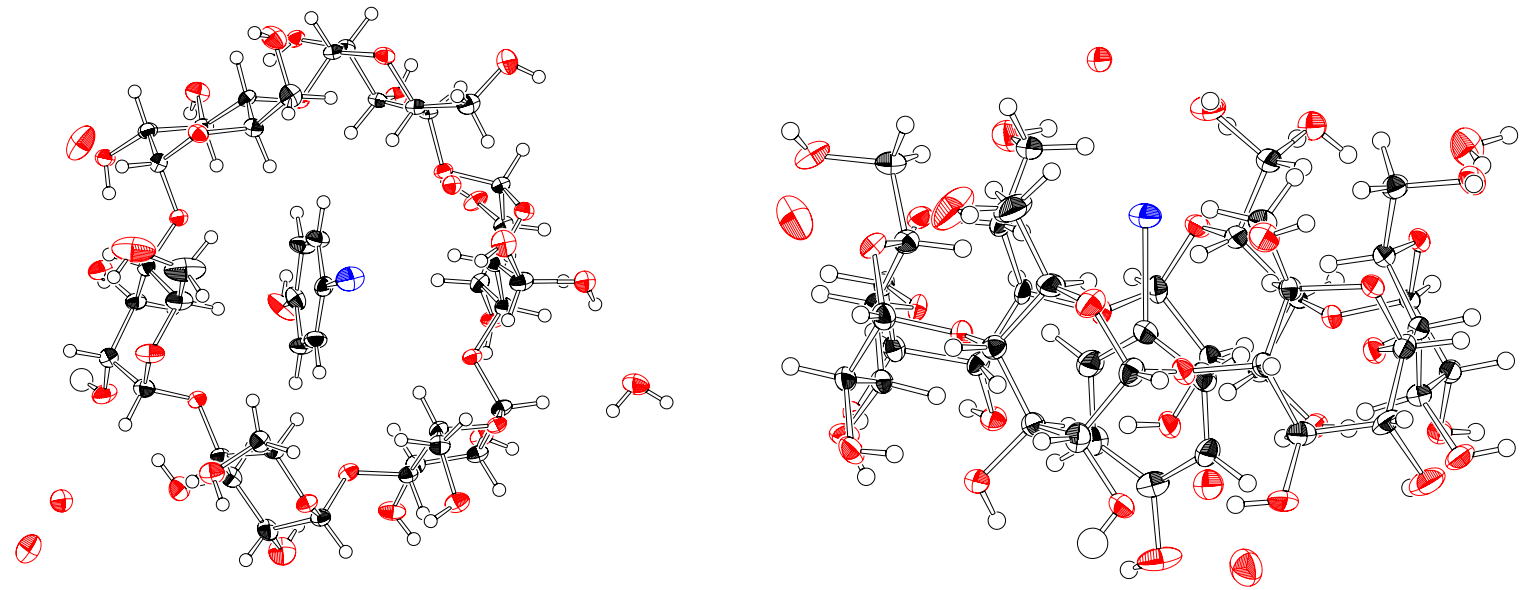

ORTEP drawing of $\alpha$-CD-4-iodophenol complex 
Crystal data for $\alpha$-CD-4-iodophenol complex

$\begin{array}{ll}\text { Chemical formula } & \mathrm{C}_{42} \mathrm{H}_{67} \mathrm{IO}_{34} \\ \text { Moiety formula } & \mathrm{C}_{36} \mathrm{H}_{60} \mathrm{O}_{30}, \mathrm{C}_{6} \mathrm{H}_{5} \mathrm{IO}, \mathrm{H}_{2} \mathrm{O}, 2(\mathrm{O}) \\ \text { MW } & 1242.86 \\ \text { Crystal system, space group } & \text { Orthorhombic, } \mathrm{P}_{2}{ }_{2}{ }_{1} 2_{1} \\ \text { Temperature }(\mathrm{K}) & 93 \\ a, b, c(\AA), V\left(\AA^{3}\right) & 13.4918(3), 15.3184(3), 24.5367(5), 5071.05(16) \\ Z & 4 \\ F(000) & 2576.00 \\ \text { Radiation type } & \mathrm{Cu} K \alpha(\lambda=1.54187 \AA) \\ \mu\left(\mathrm{mm}^{-1}\right) & 5.91 \\ \text { Crystal size }(\mathrm{mm}), \text { and color } & 0.12 \times 0.11 \times 0.10, \text { prism, colorless }\end{array}$

Data collection

Diffractometer

Absorption correction

\section{Rigaku R-AXIS RAPID}

Multi-scan

ABSCOR (Rigaku, 1995)

$T_{\min }, T_{\max }$

$0.393,0.554$

No. of measured, independent, and

observed $\left[F^{2}>2.0 \sigma\left(F^{2}\right)\right]$ reflections

$57025,9059,8624$

$R_{\text {int }}$

0.065

$(\sin \theta / \lambda)_{\max }\left(\AA^{-1}\right)$

0.6024

Refinement

$\mathrm{R}\left[F^{2}>2 \sigma\left(F^{2}\right)\right], w R\left(F^{2}\right), S$

$0.045,0.127,1.04$

No. of reflections

9059

No. of parameters

731

$\mathrm{H}$-atom treatment

$\Delta \rho_{\max }, \Delta \rho_{\min }\left(\mathrm{e} \AA^{-3}\right)$

$\mathrm{H}$ atoms treated by a mixture of independent and constrained refinement

$1.45,-1.15$

Flack x parameter ${ }^{3}$ (Parsons quotients $\left.=3392\right)$

$0.022(3)$ 


\section{a-CD-4-iodoaniline complex}

The non-hydrogen atoms and the non-disordered oxygen atoms were refined anisotropically, and the disordered oxygen atoms with high and low occupancy were refined anisotropically and isotropically, respectively. The hydrogen atoms other than the amino group and waters were refined using the riding model. The hydrogen atoms bonded to the anisotropic oxygen atoms and nitrogen atom were located on a difference Fourier synthesis, and the hydrogen atoms to the isotropic oxygen atoms were not located. The bond lengths of $\mathrm{N}-\mathrm{H}$ and $\mathrm{O}-\mathrm{H}$ of water and the $\mathrm{H}-\mathrm{O}-\mathrm{H}$ angles were ideally restrained.
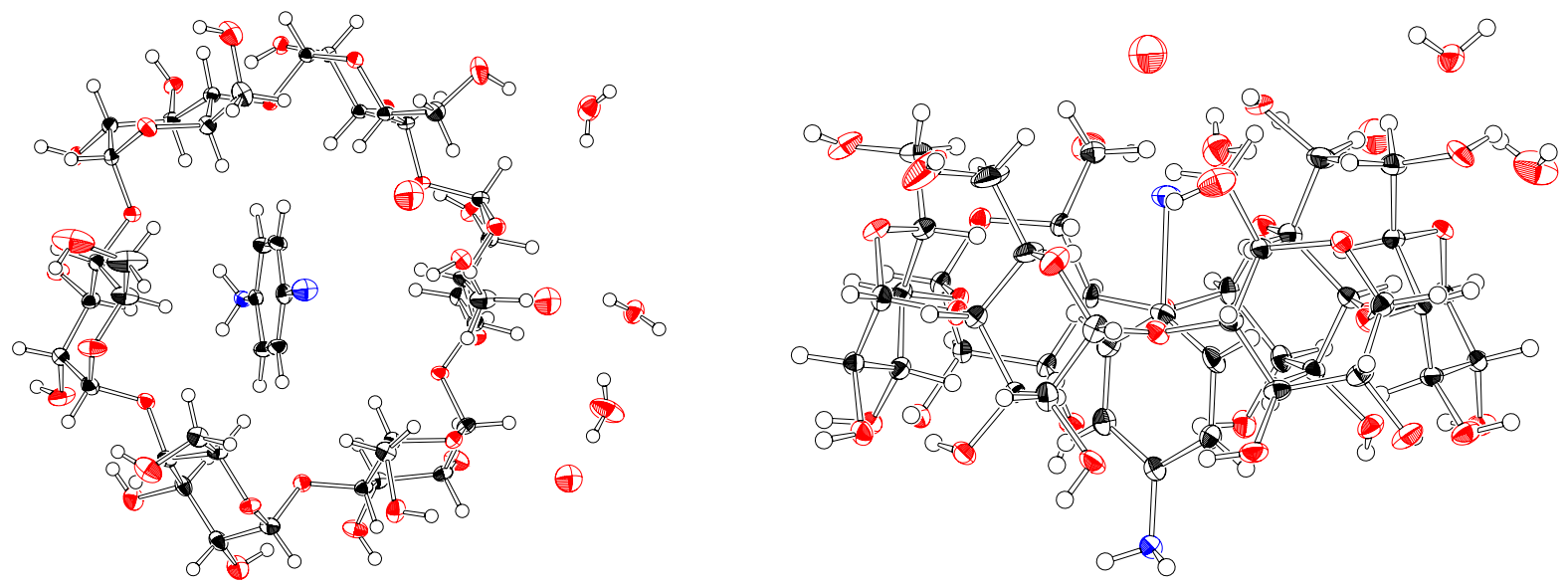

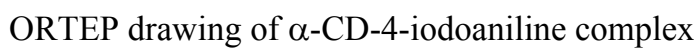


Crystal data for $\alpha$-CD-4-iodoaniline complex

$\begin{array}{ll}\text { Chemical formula } & \mathrm{C}_{42} \mathrm{H}_{78} \mathrm{~N}_{2} \mathrm{O}_{38} \\ \text { Moiety formula } & \mathrm{C}_{36} \mathrm{H}_{59.65} \mathrm{O}_{30}, \mathrm{C}_{6} \mathrm{H}_{5} \mathrm{IN}, 2.451\left(\mathrm{H}_{2} \mathrm{O}\right), 0.549(\mathrm{O}) \\ \text { MW } & 1244.46 \\ \text { Crystal system, space group } & \text { Orthorhombic, } \mathrm{P}_{1}{ }_{2} 2_{1} \\ \text { Temperature }(\mathrm{K}) & 93 \\ a, b, c(\AA), V\left(\AA^{3}\right) & 13.6848(3), 15.3807(3), 24.4482(4), 5145.90(16) \\ Z & 4 \\ F(000) & 2586.20 \\ \text { Radiation type } & \mathrm{Cu} K \alpha(\lambda=1.54187 \AA) \\ \mu\left(\mathrm{mm}{ }^{-1}\right) & 5.81 \\ \text { Crystal size }(\mathrm{mm}), \text { and color } & 0.25 \times 0.20 \times 0.16, \text { prism, colorless }\end{array}$

Data collection

Diffractometer

Absorption correction

\section{Rigaku R-AXIS RAPID}

Multi-scan

ABSCOR (Rigaku, 1995)

$T_{\min }, T_{\max }$

$0.230,0.395$

No. of measured, independent, and

observed $\left[F^{2}>2.0 \sigma\left(F^{2}\right)\right]$ reflections

58266, 9128, 8805

$R_{\text {int }}$

0.038

$(\sin \theta / \lambda)_{\max }\left(\AA^{-1}\right)$

0.6020

Refinement

$\mathrm{R}\left[F^{2}>2 \sigma\left(F^{2}\right)\right], w R\left(F^{2}\right), S$

$0.029,0.082,1.03$

No. of reflections

9128

No. of parameters

747

H-atom treatment

$\Delta \rho_{\max }, \Delta \rho_{\min }\left(\mathrm{e} \AA^{-3}\right)$

$\mathrm{H}$ atoms treated by a mixture of independent and constrained refinement

$0.94,-0.64$

Flack x parameter ${ }^{3}($ Parsons quotients $=3535) \quad 0.007(2)$ 


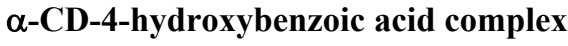

The non-hydrogen atoms were refined anisotropically. The hydrogen atoms other than waters were refined using the riding model. The hydrogen atoms bonded to non-disordered water were could located on a difference Fourier synthesis, however the hydrogen atoms bonded to disordered waters could not be located. The bond lengths and angles of water were ideally restrained.

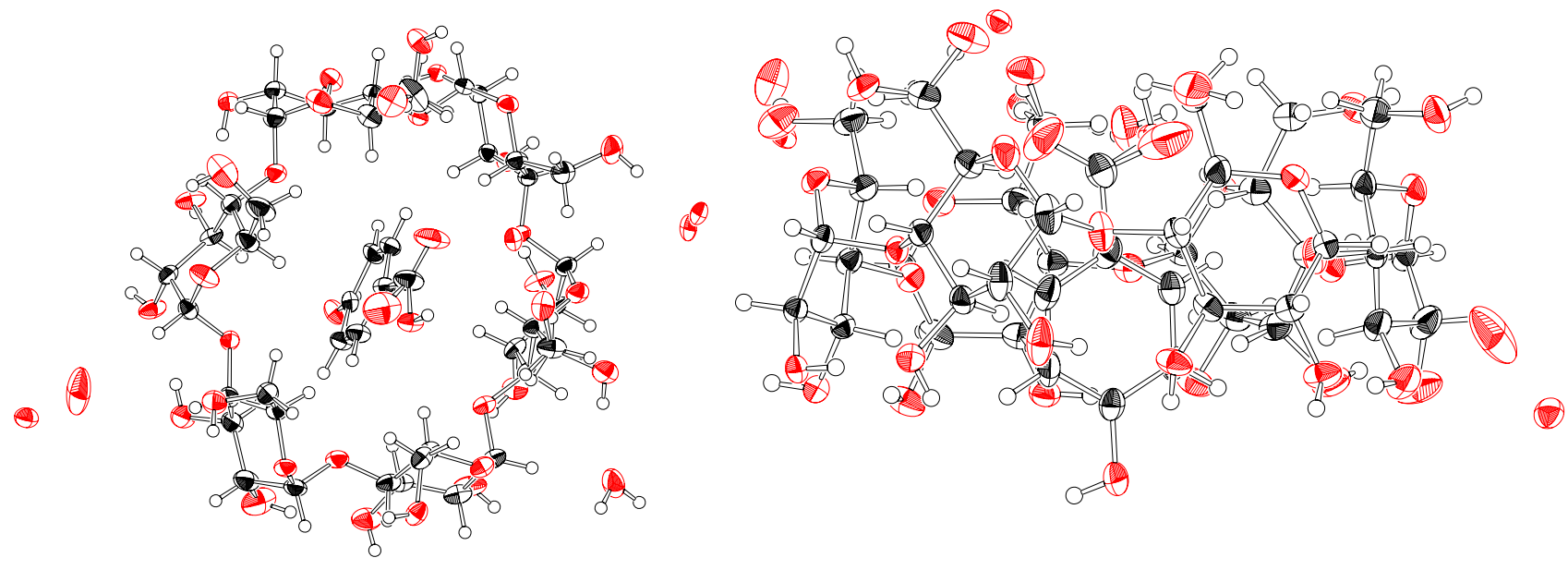

ORTEP drawing of $\alpha$-CD-4-hydroxybenzoic acid complex 
Crystal data for $\alpha$-CD-4-hydroxybenzoic acid complex

Chemical formula

Moiety formula

MW

Crystal system, space group

Temperature $(\mathrm{K})$

$a, b, c(\AA), V\left(\AA^{3}\right)$

Z

$F(000)$

Radiation type

$\mu\left(\mathrm{mm}^{-1}\right)$

Crystal size (mm), and color

Data collection

Diffractometer

Absorption correction

$T_{\min }, T_{\max }$

No. of measured, independent, and

observed $\left[F^{2}>2.0 \sigma\left(F^{2}\right)\right]$ reflections

$R_{\text {int }}$

$(\sin \theta / \lambda)_{\max }\left(\AA^{-1}\right)$

Refinement

$\mathrm{R}\left[F^{2}>2 \sigma\left(F^{2}\right)\right], w R\left(F^{2}\right), S$

No. of reflections

No. of parameters

$\mathrm{H}$-atom treatment

$\Delta \rho_{\max }, \Delta \rho_{\min }\left(\mathrm{e} \AA^{-3}\right)$

Flack x parameter ${ }^{3}($ Parsons quotients $=3900)$
$\mathrm{C}_{43} \mathrm{H}_{67.11} \mathrm{O}_{36}$

$\mathrm{C}_{36} \mathrm{H}_{59.40} \mathrm{O}_{30}, \mathrm{C}_{7} \mathrm{H}_{5.71} \mathrm{O}_{3}, \mathrm{H}_{2} \mathrm{O}, 2(\mathrm{O})$

1160.09

Orthorhombic, $\mathrm{P} 2{ }_{1} 2_{1} 2_{1}$

93

13.3368(2), 15.2925(3), 24.8268(5), 5063.50(16)

4

2452.44

$\mathrm{Cu} K \alpha(\lambda=1.54187 \AA)$

1.18

$0.12 \times 0.09 \times 0.09$, prism, colorless

Rigaku R-AXIS RAPID

Multi-scan

ABSCOR (Rigaku, 1995)

$0.735,0.899$

59196, 9230, 9017

0.023

0.6018

$0.042,0.121,1.06$

9230

790

$\mathrm{H}$ atoms treated by a mixture of independent and constrained refinement

$0.82,-0.27$

$0.05(2)$ 


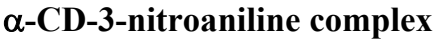

The non-hydrogen atoms were refined anisotropically. The hydrogen atoms other than waters were refined using the riding model. The hydrogen atoms bonded to water were could located on a difference Fourier synthesis. The bond lengths and angles of water were ideally restrained.
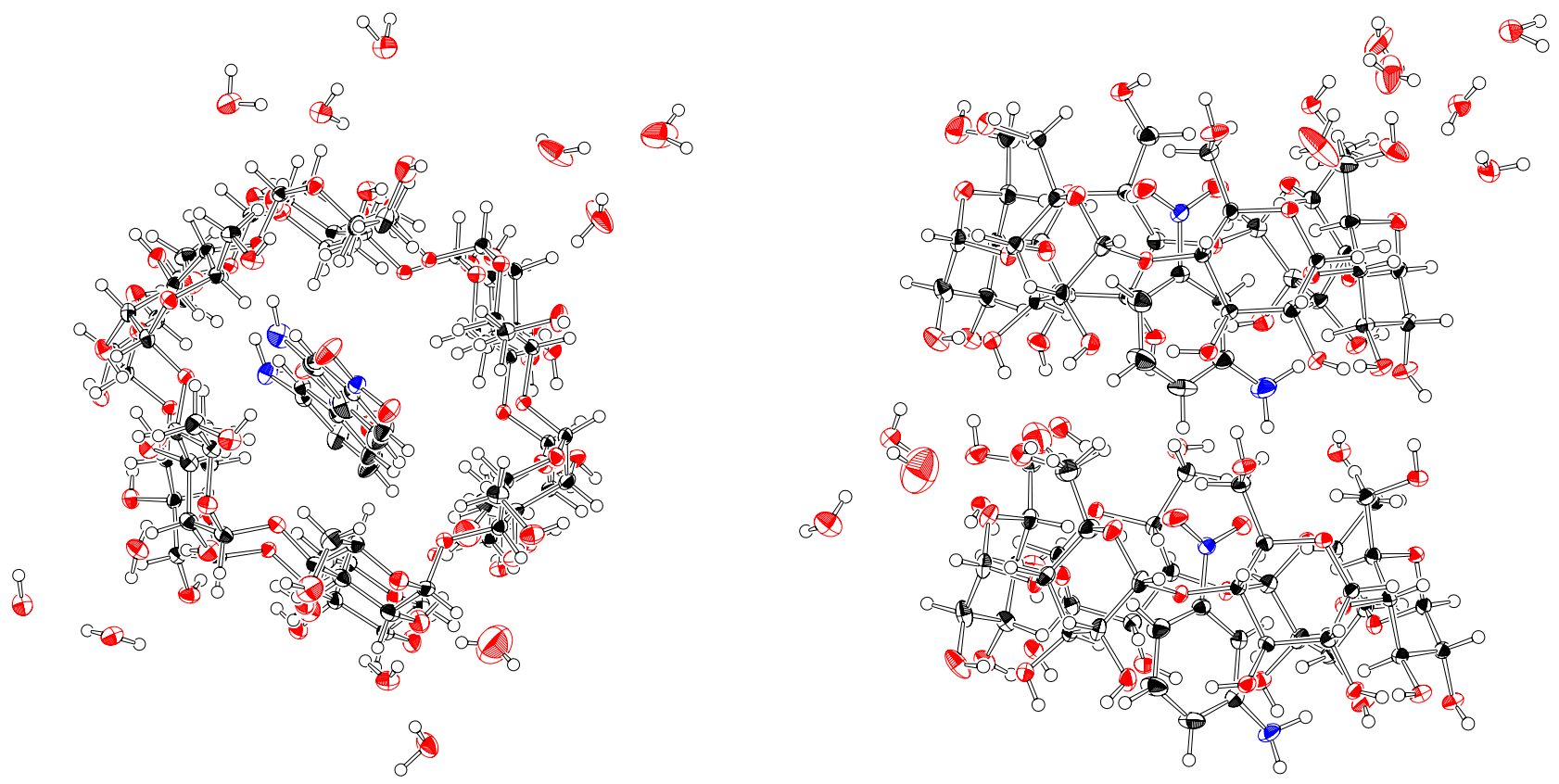

ORTEP drawing of $\alpha$-CD-3-nitroaniline complex 
Crystal data for $\alpha$-CD-3-nitroaniline complex

$\begin{array}{ll}\text { Chemical formula } & \mathrm{C}_{42} \mathrm{H}_{78} \mathrm{~N}_{2} \mathrm{O}_{38} \\ \text { Moiety formula } & \mathrm{C}_{36} \mathrm{H}_{60} \mathrm{O}_{30}, \mathrm{C}_{6} \mathrm{H}_{6} \mathrm{~N}_{2} \mathrm{O}_{2}, 6\left(\mathrm{H}_{2} \mathrm{O}\right) \\ \text { MW } & 1219.07 \\ \text { Crystal system, space group } & \text { monoclinic, } \mathrm{P}_{1} \\ \text { Temperature }(\mathrm{K}) & 93 \\ a, b, c(\AA), V\left(\AA^{3}\right) & 16.0055(3), 13.4884(3), 25.3797(5) \\ \beta\left({ }^{\circ}\right), \text { and } V\left(\AA^{3}\right) & 104.127(7), 5313.5(2) \\ Z & 4 \\ F(000) & 2592.00 \\ \text { Radiation type } & \mathrm{Cu} K \alpha(\lambda=1.54187 \AA) \\ \mu\left(\mathrm{mm}^{-1}\right) & 1.19 \\ \text { Crystal size }(\mathrm{mm}), \text { and color } & 0.20 \times 0.15 \times 0.10, \text { prism, orange }\end{array}$

Data collection

Diffractometer

Absorption correction

Rigaku R-AXIS RAPID

Multi-scan

ABSCOR (Rigaku, 1995)

$T_{\min }, T_{\max }$

$0.698,0.893$

No. of measured, independent, and

observed $\left[F^{2}>2.0 \sigma\left(F^{2}\right)\right]$ reflections

$61744,18671,18434$

$R_{\text {int }}$

0.0 .025

$(\sin \theta / \lambda)_{\max }\left(\AA^{-1}\right)$

0.6021

Refinement

$\mathrm{R}\left[F^{2}>2 \sigma\left(F^{2}\right)\right], w R\left(F^{2}\right), S$

$0.039,0.110,1.03$

No. of reflections

18671

No. of parameters

1601

$\mathrm{H}$-atom treatment

$\mathrm{H}$ atoms treated by a mixture of independent and constrained refinement

$\Delta \rho_{\max }, \Delta \rho_{\min }\left(\mathrm{e} \AA^{-3}\right)$

$0.51,-0.58$

Flack x parameter ${ }^{3}$ (Parsons quotients $\left.=8162\right)$

$0.06(2)$

\section{References}

1) Sheldrick, G. M. Acta Cryst. 2014, A70, C1437.

2) Sheldrick, G. M. Acta Cryst. 2008, A64, 112-122.

3) Parsons, S.; Flack, H. D.; Wagner, T., Acta Cryst. 2013, B69, 249-259. 